\title{
AnyCasting In Dual Sink Approach (ACIDS) for WBASNs
}

\author{
Muhammad Rahim Baig \\ Department of Computer Science \\ CECOS University of IT and Emerging Sciences \\ Peshawar, Pakistan \\ Preston University, Peshawar, Pakistan
}

\author{
Najeeb Ullah \\ Department of Computer Science \\ CECOS University of IT and Emerging Sciences \\ Peshawar, Pakistan
}

\author{
Fazle Hadi \\ Department of Computer Science \\ Preston University, Peshawar, Pakistan
}

\author{
Sheeraz Ahmed \\ Department of Electrical Engineering \\ Gomal University \\ Dera Ismail Khan, Pakistan \\ CECOS University, Peshawar, Pakistan
}

\author{
Abdul Hanan \\ Department of Computer Science \\ CECOS University of IT and Emerging Sciences \\ Peshawar, Pakistan \\ Imran Ahmed \\ Department of Computer Science \\ Institute of Management Sciences, Peshawar, Pakistan
}

\begin{abstract}
After successful development in health-care services, WBASN is also being used in other fields where continuous and distant health-care monitoring is required. Various suggested protocols presented in literature work to enhance the performance of WBASN by focusing on delay, energy efficiency and routing. In this research we focus to increase the stability period and throughput, while decreasing end-to-delay. Two sink nodes are utilized and concept of AnyCasting is introduced. In this research, we have presented a scheme AnyCasting In Dual Sink (ACIDS) for WBASN and compared it with existing protocols LAEEBA and DARE. The performance of ACIDS is found to be $51 \%$ and $13 \%$ efficient than LAEEBA and DARE respectively in throughput. Results show that, the stability period of ACIDS is much greater than LAEEBA and DARE with minimum delay. Energy parameter in ACIDS is in tradeoff with the improved parameters, due to the computation of RSSI which does more processing and utilizes more energy.
\end{abstract}

Keywords-Stability Period; WBASNs; Throughput; End-toEnd Delay; Energy Consumption; AnyCasting; RSSI

\section{INTRODUCTION}

Computer Science is now growing rapidly to operate large data and keep higher level of linking. At the current era advancement also occur in small scale networks which support higher level of access and mobility [1], [2]. Wireless Body Area Sensor Networks (WBASNs) is a new field of Wireless Sensor Networks (WSNs), started developing since 1995 [3]. The objective of this network is to establish communication associated to human body. Initially it was developed for the purpose of providing health-care services to serious patient. After the successful development in health-care services now WBASNs is also being used in other fields where continuous and distant health-care monitoring is necessary, like, players in sports, astronaut in space, environmental condition, motion detection of animals, security, etc. [4], [5].

WBASNS consists of small size sensors which have minimum energy source and processing capability. Depending upon the application, these sensors can be fixed inside the body or can be wearable sensors attached on body [2].

Data rate and power consumptions of implanted devices are low as compared to that of wearable devices [6], [7]. In medical field, different sensors are attached to human body that is residing at home or hospital. These sensor nodes detect and monitor different biological parameters of human body like glucose-rate, Electrocardiogram (ECG) temperature, heart-rate, blood-rate, heart beat and blood pressure etc. [8]. The received signals are aggregated or collected by a personal device, e.g. Personal Digital Assistance (PDA) which, act as a relay node and sent them to health treatment professionals or to some internet using applications with the help of sink node. The sink acts as a source of route between the hospital, and the WBASNs [9].

In WBASNs, sensor nodes are battery driven units that run with limited energy source [7]. It is necessary to use minimum energy for transmitting data from sensor nodes to sink. One of the major task in this network is to recharge the batteries of senor nodes, therefore it is demanded for energy efficient protocol which lessen the issue of recharging the batteries. In [4], researchers found some challenges faced by WBASNs which are as follows:

- Trade-off between communication and processing

- Bandwidth and power consumption

- High level of attenuation as compared to other WSNs applications 


\section{- Storage and energy harvesting}

Usually, in WBASNs there is sink that collects data from the battery-powered sensor nodes and use unicast transmission to transmit the sensed data to the sink node; in transmission every sensor node has only one destination, which is a single sink. Every sensor nodes sense data and forward to this single sink node. Problems may arise while using single sink and transmitting data through unicasting, which are discussed in detail in letter section. Another way of using two sinks is a well approach in WSNs; in two sinks approach all the sensors in the sensing area sense the environmental information and forward to any available sink node in AnyCast manner [10]. AnyCasting is a best working technology in WSNs and none of researchers use this technology in WBASNs.

WBASNs, in the current era of sensor networks is the hot area of research, where, much more papers have been published on Quality of Service (QoS) energy efficiency, bandwidth and security, the topology of nodes in a body and transmission of data between the sensors and the sink is the focusing point. Therefore, in this study we are going to address these issues by considering the transmission technique.

Rest of the paper is further divided into sub sections which are described as follows. Section II provides some existing works. In section III motivation behind this research is mentioned. Proposed model for ACIDS protocol is presented in Sections IV. Simulation results are discussed in section $\mathrm{V}$ and finally the paper is concluded in section VI.

\section{RELATED WORK}

Different researchers have proposed different protocols and techniques to increase performance and reliable communication. Some of them are summarized and discussed below.

In paper [1], authors propose Incremental relay-based CoCEStat protocol for Wireless Body Area Networks (InCoCEStat). Author's uses two relay nodes for cooperation purpose to quick transmit the critical data in emergency cases in WBASNS. Relay nodes detects the data from sensors and forward to the single destination sink node in three phases with a dublicat copy to decrease the chance of packet drop. Researchers in [2], propose a protocol called DARE. This protocol is used to monitor eight patients in a ward, each comprises of seven sensors to monitor different parameters of the patient body. Five topological scenarios have been proposed where more than one sink nodes are placed in different location of the ward.

In [3], authors present a protocol name as SIMPLE. In this protocol, author uses eight sensors and single sink node in human body. Nodes lose enery in short span of time therfore, to balance energy level and stability period of the network a mathematical formula is suggested to select cluster head/forwarder in a cluster. In paper [5], authors present a protocol; THE-FAME (Threshold based Energy-efficient Fatigue MEasurment) to find fatigues of a player in a Soccer game. Each player is implanted a sensor node to sense fatigue's parameter of the player. Multiple sink nodes are attached to the different side of the game. The size of sensor kept small, sensor send data through direct communication when threshold level is reached.

Researchers in [6], present hardware and software architecture, error detection and data transmission for normal and emergency data. Authors used a cluster head $(\mathrm{CH})$ to gather the parametric data. Researchers in [8] present another protocol called Effect of Packet Inter-arrival Time on the Energy Consumption of Beacon Enable MAC Protocol for WBASNS. In this protocol, researchers focus on Media Access Control (MAC) to reduce the energy consumption. Two type of nodes has been used; Fully Functional Devices (FFD) and Reduced Functional Devices (RFD). FFD can be simple node or coordinator node and RFD works only as simple node.

In [7], a protocol called LAEEBA is proposed for Wireless Body Area Networks. In this scheme authors uses both direct communication and multi-hop communication by focusing the path loss. Cost function is calculated to minimize the energy consumption. In paper [9], a protocol called M-ATTEMPT is proposed, which is threshold based routing protocol to find the link spot in the established links. In this model authors placed the sensors in decreasing order of their data rate with respect to the sink. Both single hope and multi hope communication is used for emergency and normal data. In [11], S. Ahmad et al. revised their previous work (LAEEBA), and propose new routing protocol for WBASNS named as Co-LAEEBA. New protocol is suggested for collective working and path loss factors. Knowing the residual energy and sharing the distance from sensor to the sink, cost function is introduced to select the feasible route to the destination.

In paper [12], researchers propose a routing protocol that focuses the accessibility of patient's data either by offline or online to the health care takers. According to the propose model different sensors are placed at the patients cloths (on body). Data are gathered by the accumulator and send to the medical server through Wi-Fi. Researchers in [13], take four attributes into accounts which are; distance covered, residual energy, hop-count and node criticality. Routing occur in two phase i.e. setup and operational phase. In paper [14], cost based routing protocol is proposed for Wireless Body Area Networks" is proposed. In this paper energy efficiency and cost function is focused by considering the reliability of path on basis on critical factor. In paper [15], researchers used two existing protocol: Dynamic Source Routing (DSR), and Energy efficient self-adaptive route E-DSR with tree topology to minimize energy consumption and network lifecycle as. In paper [16], authors present a protocol which improves the throughput and avoid single point of failure in WBASNS. The protocol uses Cooperative Network Coding (CNC) in many to many as multiple input multiple output (MIMO). Researchers in [17], propose a robust and fast routing protocol focusing on topological design. Because of the heterogeneity of sensor nodes and their energy consumption researcher takes advantage of suitable linear relaxations to guide a randomized fixing of the variables, supported by large variable neighborhood search. This algorithm also focuses the traffic uncertainty in the design of WBASNS by using relay nodes, and use preferably single path routing. 
In paper [19], authors propose a mathematical technique that uses network topology and cross-layer optimization in WBSNs. Authors introduce multilevel primal and dual decomposition methods to solve the non- convex mixedinteger optimization problem.

Authors in [20] design a network architecture that uses WBASNS and Cloud for the purpose of sharing data. Data sharing in this model occurs in four different layers with the help of TCP/IP and Zigbee. In-order to support mobility of users, WBAN coordinators inter-operates with different local networks such as WiFi and LTE. Furthermore, adaptive streaming technique is also used in this paper to reduce packet loss.

\section{Motivation}

According to the literature survey, majority of the researchers [1], [3], [7]-[11] used single sink node to aggregate and send the sense data from the sensor nodes to the destination. Single sink node collects data from all sensors and forward to the destination. Some problems may arise for which the researchers did not focus which are: first, the overall topology will fail when the single sink node die or stop working for some time for any reason. Second, all sensors send their data to this sink which makes an over burden for it to aggregate and quick delivery. Over burden problem leads to delay between packets, especially for emergency data which needs quick delivery. The performance factor increase when more number of packets will send in less time and received error free to the medical caretaker. This over burden on sink leads to decrease in delivery ratio.

In [17], authors presented a new scheme to make fast and robust design for WBASNS. In this scheme authors prefer to use a greater number of relay nodes in the body, almost equal to normal nodes, leads to increase the network cost creates delay and decrease delivery ratio, which the author did not focused. The placement of sink in exact location by considering the LoS and NLoS is important. Misplacement of sink node leads to disconnection of sensor from the sink node when the body parts move. According to the figure representation correct placement of sinks are not mentioned. In [14], author placed the sink at wrist that leads to disconnect the previously established link when the arm moves back and forth due to NLoS. To overcome the limitation in above discussion we proposed ACIDS scheme for WBASNs. In ACIDS, the performance is increasing by taking care of delivery ratio, stability period and end-end-delay.

\section{ACIDS PROPOSED PROTOCOL}

In this section, we presents our proposed routing protocol ACIDS for WBASNS with AnyCasting technique to improve the performance of routing scheme by using two sinks.

\section{A. Network Topology}

In ACIDS we placed two sinks named as S1 and S2 with eight sensors. S1 is placed at right lumbar and S2 is at left lumbar to consider NLoS communication in case of body parts movement i.e. arm. Both cases of moving back and forth the arm is in LoS communication with lumbar, therefore it is a suitable location we selected. Node 1 and 4 transmit data direct to S1 and S2 in order to consider the NLoS issue (as discussed above). The other nodes which are in direct range to either sinks transmit data in direct communication and the rest nodes transmit through multi-hop communication by selecting a forwarder node. Topology of our proposed protocol ACIDS is mentioned in figure 1.

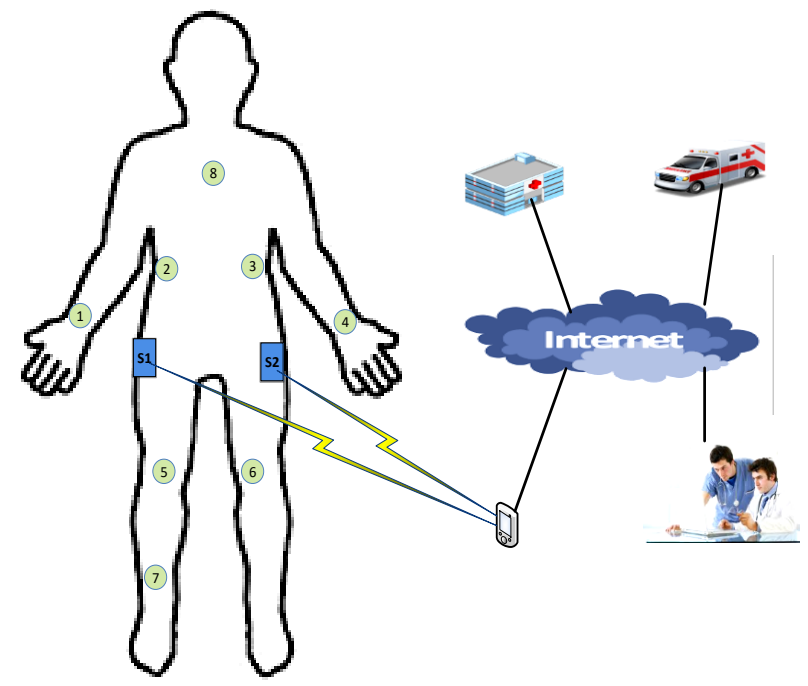

Fig. 1. Schematic diagram for ACIDS Protocol

\section{B. Initialization Phase}

In this phase, two sinks initially broadcast a "Hello" message to all sensor nodes that contains Sinks Id and location. Receiving this message sensor nodes save location and Id of Sinks in their routing table. Sensor nodes also broadcast some packets that contain the node Id, node energy level and their location. In this way each node is aware about their neighbors.

\section{Selection of Next-Hop Phase}

ACIDS computes forwarder node on the basis of threshold Residual Energy (RE). ACIDS fix a threshold value of RE is equal to $0.1 \mathrm{~J}$.

$$
\text { RE }>=\text { Threshold } \ldots . . . \text { where, Threshold }=0.1 \mathrm{~J} \text {. }
$$

Here, if more than one nodes RE greater than threshold, than forwarder node will be selected through Received Signal Strength Identification (RSSI), [18]. RSSI describe relation between the received power and the transmitted power of wireless signal and the distance among them. ACIDS follow the RSSI model of [19] that is mentioned below in equation (2).

$$
\operatorname{Pr}=\operatorname{Pt} \cdot\left(\frac{1}{d}\right)^{n}
$$

Here, $\mathrm{Pr}$, is the wireless signal received power. Pt, shows the power transmitted by the wireless signal, and distance between sending and receiving node is denoted by $d$. Transmission factor between sending and receiving node is denoted by $\mathrm{n}$, whose value depends on propagation 
environment. According to this equation, the node is selected for forwarder node whose $\mathrm{Pr}$ value is maximum.

\section{TABLE I. SimULATION PARAMETERS USED}

\begin{tabular}{|l|l|}
\hline Parameters & Values \\
\hline DC current (RX) & $18 \mathrm{~mA}$ \\
\hline DC current (TX) & $10.5 \mathrm{~mA}$ \\
\hline Minimum supply voltage & $1.9 \mathrm{~V}$ \\
\hline ERr & $36.1 \mathrm{~nJ} / \mathrm{bit}$ \\
\hline ERt & $16.7 \mathrm{~nJ} / \mathrm{bit}$ \\
\hline ERamp & $1.97 \mathrm{~nJ} / \mathrm{bit}$ \\
\hline Wavelength $(\lambda)$ & $0.135 \mathrm{~m}$ \\
\hline Frequency (f) & $2.5 \mathrm{GHz}$ \\
\hline Initial Energy (Eo) & $0.7 \mathrm{~J}$ \\
\hline do & 0.15 \\
\hline
\end{tabular}

\section{Routing and Energy Consumption Phase}

Another advantage of using two sinks is that, majority of nodes comes in direct range. In direct communication packets are delivering without any delay. According to the proposed protocol ACIDS, nodes 1 and 4 send data directly to S1 or S2 to consider the NLoS of arm movement. The nodes which are in direct range to either S1 or S2 will send data directly, other nodes AnyCast to S1 or S2 through relay node in multi-hop communication. Relay node will be selected until it comes in direct range to either sink. The communication flow of the ACIDS protocol is mentioned in figure 2 .

Energy consumption in multi-hop $\left(\mathrm{E}_{\mathrm{m}}\right)$ communication is mentioned below [5]:

$$
\begin{aligned}
& \mathrm{ER}_{\mathrm{t}-\mathrm{m}}(\mathrm{k}, \mathrm{d})=\mathrm{n} \times\left(\mathrm{ER}_{\mathrm{cr}}+\mathrm{ER}_{\mathrm{amp}}\right) \times \mathrm{k} \times \mathrm{d}^{2} . \\
& \mathrm{ER}_{\mathrm{r}-\mathrm{m}}(\mathrm{k})=(\mathrm{n}-1) \times\left(\mathrm{ER}_{\mathrm{cr}}+\mathrm{ER}_{\mathrm{amp}}\right) \times \mathrm{k} \ldots \ldots \ldots \\
& \mathrm{ER}_{\text {total-m }}=\mathrm{ERt}-\mathrm{m}+\mathrm{ER}_{\mathrm{r}-\mathrm{m}} \ldots \ldots \ldots \ldots \ldots \ldots \ldots \ldots \ldots \ldots
\end{aligned}
$$

Energy consumption in direct $\left(\mathrm{E}_{\mathrm{D}}\right)$ communication is [5]:

$$
\begin{aligned}
& \mathrm{ER}_{\mathrm{t}-\mathrm{D}}(\mathrm{k}, \mathrm{d})=\left(\mathrm{ER}_{\mathrm{cr}}+\mathrm{ER}_{\mathrm{amp}}\right) \times \mathrm{k} \times \mathrm{d}^{2} \ldots \\
& \mathrm{ER}_{\text {total-D }}=\mathrm{ER}_{\mathrm{t}-\mathrm{D}} \ldots \ldots \ldots \ldots \ldots \ldots \ldots \ldots \ldots \ldots \ldots \ldots
\end{aligned}
$$

Here, $E_{t}$ and $E R_{r}$ are the energy required for transmission and receive by sender and receiver. In equation $\mathrm{k}$, represent the size of bits and $\mathrm{d}$, is the distance between nodes and sinks. $\mathrm{ER}_{\mathrm{cr}}$ is the required energy to run the electronic circuit of the receiver and transmitter. $\mathrm{ER}_{\mathrm{amp}}$ is the required energy to amplify $\mathrm{k}$ bits to the distance $\mathrm{d}$. in the above equation 3 and 4 $\mathrm{n}$ is the number of nodes needed to reach the sinks and $\mathrm{d}^{2}$, is the loss of energy while transmitting data through transmission channel. The energy parameters depend on the hardware used. Energy parameters used in ACIDS are mentioned in the table 1 .

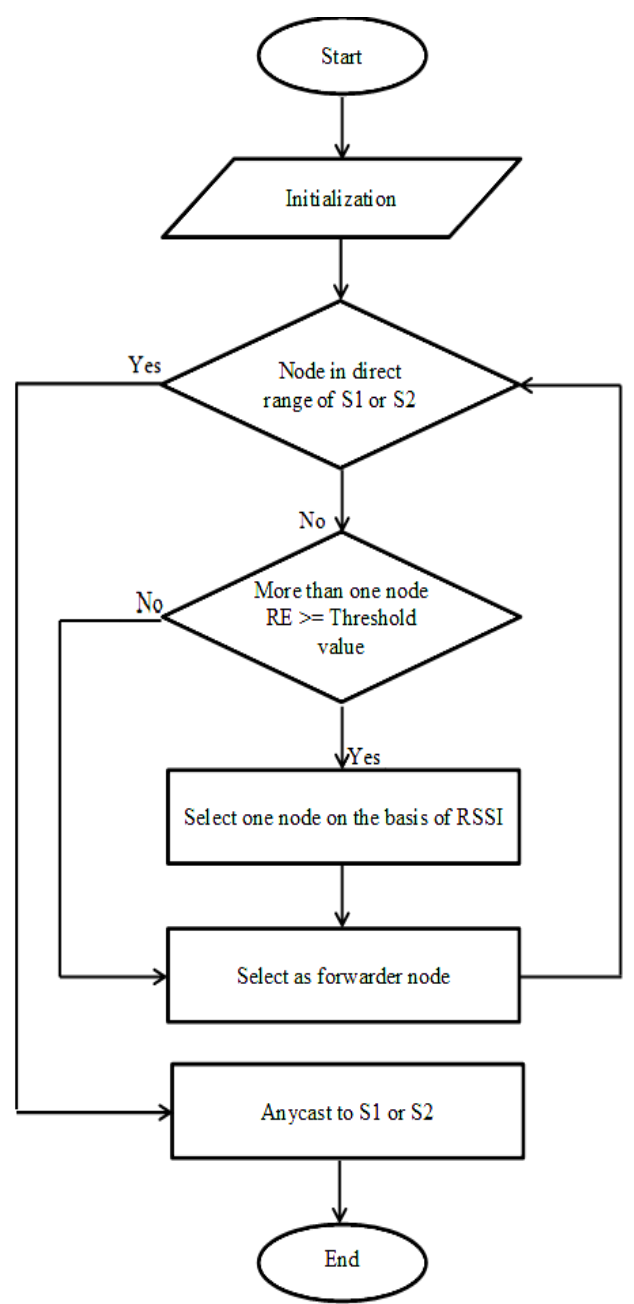

Fig. 2. Flow Chart for ACIDS Protocol

\section{RESULTS AND DISCUSSIONS}

In this paper we have conducted a series of simulations to measure the performance of our proposed protocol ACIDS. We compared it with the existing protocols: DARE and LAEEBA. All three protocols are evaluated for same key performance parameters: Stability Period, End-to-End Delay, Throughput and residual energy. Energy Parameter used in simulations is enlisted in table 1 .

\section{A. Stability Period}

Stability Period is the time duration of operation of network till the first node dies. According to figure 3, performance of ACIDS is much better than LAEEBA and DARE. Table 2 shows the numerical values of dead nodes for all the three protocols starting from time 1000 till time 10000. First node of ACIDS dies at time 7200, while LAEEBA loses its first node at time 2100 and DARE at time 4250. The 
increased performance of ACIDS is because of availability of more than one sink. Each sink comes near to nodes which helps them to send data to the nearest sink node. Another obvious reason for better performance is the location of sinks. The wrist sensors nodes direct communicate to S1 and S2 without NLoS issue. These factors also improve the performance of ACIDS up to the 10000 times. All nodes of LAEEBA and DARE are dead at time 8000, while last node of ACIDS dead in time 10000.

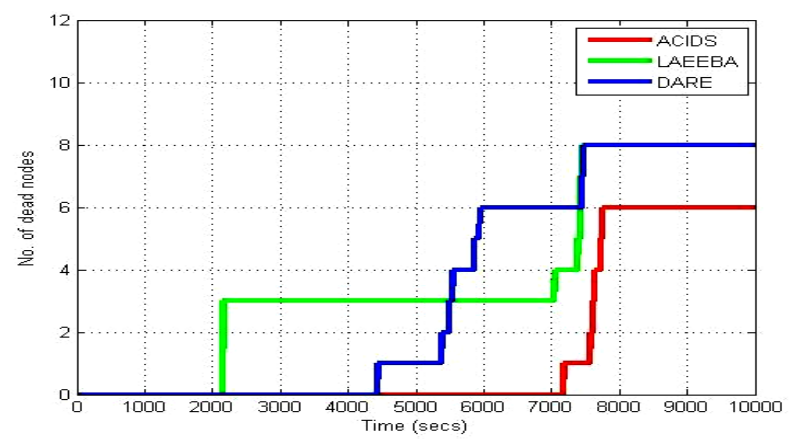

Fig. 3. Stability Period vs Time

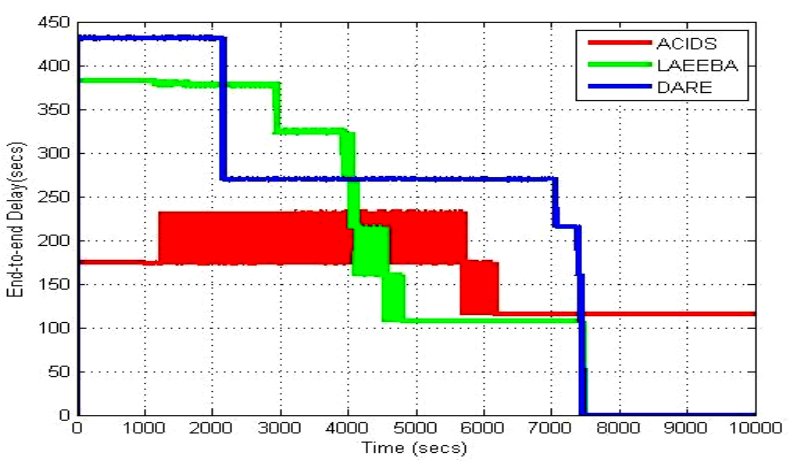

Fig. 4. End-to-End Delay vs Time

\section{B. End-to-End Delay}

The term delay refers to the time lag between the source nodes and the destination sinks. Minimizing delay in WBASNS is an important factor. The data sense from the sensors is to be evaluated by medical specialists, so it is important to receive data in time. It will be obtained if the sinks gather data in time. Figure 4 shows that the performance of ACIDS protocol, as compared to LAEEBA and DARE protocol is much better. The average delay values are mentioned in table 3, which shows that ACIDS takes an average of 162.34, which is $27 \%$ efficient than DARE. LAEEBA takes an average of 167.97. The evaluated percentage value in table 5.2 shows that the ACIDS is $2 \%$ efficient than LAEEBA. The achieved performance is due to the availability of AnyCasting between two sink nodes. The sensor nodes have two option to send their data. The LAEEBA and DARE protocol uses single sink which waste their time in calculation for gathering data from more than one sensor nodes. The availability of two sink in ACIDS makes its performance better because each sink gather few of the sensors data. Another valid reason for achieving better performance as compared to LAEEBA and DARE is: majority of the sensors in ACIDS comes in direct communication to either $\mathrm{S} 1$ or $\mathrm{S} 2$ and the distance between sensors and sink become lessen. It is obvious from the literature survey that delay will decrease in direct communication as compared to Multi-hop communication if the distance is lessens.

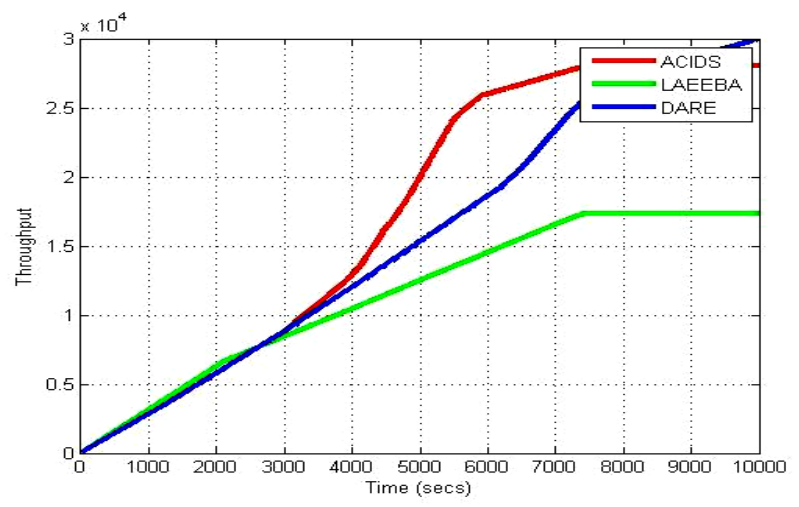

Fig. 5. Throughput vs Time

\section{Throughput}

Throughput is the successful delivery of packets from sensors to the sinks in per unit time. The unit here is taken as second starts from 1000 to 10000 . Two type of links used in ACIDS one is from sensors to the forwarder node and one is from forwarder to sinks. The link from forwarder to the sink transmits more data as compared to the other link. So, in ACIDS more number of sensors come near to sinks, which transmit data directly, which leads to increase the throughput. Figure 5, shows a clear difference in performance among three protocols. Results show that the performance of ACIDS is better than that of other two. The average throughput value of ACIDS according to table 4 is 18790 , which is $51 \%$ better than LAEEBA and $13 \%$ than DARE.

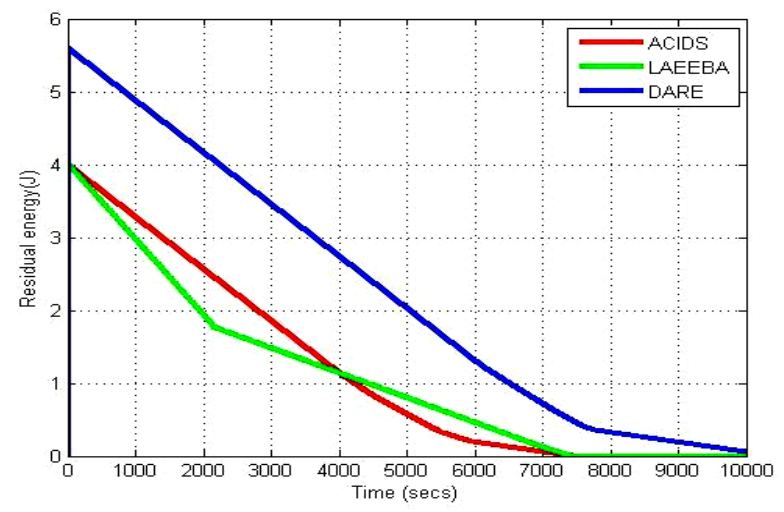

Fig. 6. Energy Consumption vs Time

\section{Net Energy Consumption}

For clear evaluation, we kept same initial energy for all protocols that is 0.7 Joule. According to the figure 6 and table 5 , it shows that the energy level of ACIDS protocol is better than LAEEBA protocol but not efficient than DARE protocol. The energy parameter in ACIDS comes in tradeoff with delay, throughput and stability period. In ACIDS our aim was to improve above said parameters. We proved our aim by 
showing efficient results in these parameters. However, ACIDS shows an improved performance as compared to LAEEBA.

The improved performance of DARE in energy is just because that DARE is focused to improve the energy parameter of WBASNS, in ACIDS we focused to improve the throughput, end-to-end delay and stability period. Another reason of depletion in energy is the use of RSSI in ACIDS. Our proposed protocol ACIDS do extra calculation to find the forwarder node when two or more nodes have the residual energy more than threshold range of energy. This extra calculation leads in depletion of energy more than DARE protocol. Tie situation in selecting the forwarder node is the focusing point, unfortunately the researchers did not focused.

TABLE II. DEAD NODES VS TIME (SECONDS)

\begin{tabular}{|l|l|l|l|l|l|l|l|l|l|l|}
\hline Protocol & $\mathbf{1 0 0 0 s}$ & $\mathbf{2 0 0 0 s}$ & $\mathbf{3 0 0 0}$ & $\mathbf{4 0 0 0 s}$ & $\mathbf{5 0 0 0 s}$ & $\mathbf{6 0 0 0 s}$ & $\mathbf{7 0 0 0 s}$ & $\mathbf{8 0 0 0 s}$ & $\mathbf{9 0 0 0 s}$ & $\mathbf{1 0 0 0 0 s}$ \\
\hline LAEEBA & 0 & 0 & 3 & 3 & 3 & 3 & 3 & 8 & 8 \\
\hline DARE & 0 & 0 & 0 & 0 & 1 & 6 & 6 & 8 & 8 \\
\hline ACIDS & 0 & 0 & 0 & 0 & 0 & 0 & 2 & 6 & 6 \\
\hline
\end{tabular}

TABLE III. END-TO-END DELAY VS TIME (SECONDS)

\begin{tabular}{|c|c|c|c|c|c|c|c|c|c|c|c|c|}
\hline \multirow{2}{*}{ Protocol } & \multicolumn{2}{|c|}{ Improvement } & \multirow{2}{*}{$1000 \mathrm{~s}$} & \multirow{2}{*}{$2000 s$} & \multirow{2}{*}{$3000 s$} & \multirow{2}{*}{$4000 \mathrm{~s}$} & \multirow{2}{*}{$5000 \mathrm{~s}$} & \multirow{2}{*}{$6000 \mathrm{~s}$} & \multirow{2}{*}{$7000 \mathrm{~s}$} & \multirow{2}{*}{8000 s } & \multirow{2}{*}{$9000 s$} & \multirow{2}{*}{$10000 \mathrm{~s}$} \\
\hline & \%Age & Average & & & & & & & & & & \\
\hline LAEEBA & $75 \%$ & 167.97 & 382.7 & 380.7 & 327.3 & 268.9 & 106.7 & 106.7 & 106.7 & 0 & 0 & 0 \\
\hline DARE & $100 \%$ & 221.52 & 431.8 & 433.3 & 269.4 & 270.7 & 270.1 & 269.3 & 270.6 & 0 & 0 & 0 \\
\hline ACIDS & $73 \%$ & 162.34 & 174.6 & 173.5 & 232.3 & 173.5 & 232.5 & 174.6 & 115.6 & 115.6 & 115.6 & 115.6 \\
\hline
\end{tabular}

TABLE IV. PACKet Delivery RATIO vs Time (SECONDS)

\begin{tabular}{|c|c|c|c|c|c|c|c|c|c|c|c|c|}
\hline \multirow{2}{*}{ Protocol } & \multicolumn{2}{|c|}{ Improvement } & \multirow{2}{*}{$1000 \mathrm{~s}$} & \multirow{2}{*}{ 2000s } & \multirow{2}{*}{$3000 \mathrm{~s}$} & \multirow{2}{*}{$4000 \mathrm{~s}$} & \multirow{2}{*}{$5000 \mathrm{~s}$} & \multirow{2}{*}{$6000 \mathrm{~s}$} & \multirow{2}{*}{$7000 \mathrm{~s}$} & \multirow{2}{*}{$8000 s$} & \multirow{2}{*}{$9000 \mathrm{~s}$} & \multirow{2}{*}{$10000 \mathrm{~s}$} \\
\hline & $\%$ Age & Average & & & & & & & & & & \\
\hline LAEEBA & $100 \%$ & 12479.8 & 3167 & 6296 & 8515 & $1.057 \mathrm{e}+04$ & $1.261 \mathrm{e}+04$ & $1.465 \mathrm{e}+04$ & $1.667 \mathrm{e}+04$ & $1.744 \mathrm{e}+04$ & $1.744 \mathrm{e}+04$ & $1.744 \mathrm{e}+04$ \\
\hline DARE & $138 \%$ & 17246.3 & 2800 & 5777 & 8806 & $1.199 \mathrm{e}+04$ & $1.532 \mathrm{e}+04$ & $1.861 \mathrm{e}+04$ & $2.339 \mathrm{e}+04$ & $2.719 \mathrm{e}+04$ & $2.859 \mathrm{e}+04$ & $2.999 \mathrm{e}+04$ \\
\hline ACIDS & $151 \%$ & 18790 & 2819 & 5782 & 8829 & $1.289 \mathrm{e}+04$ & $2.004 \mathrm{e}+04$ & $2.6 \mathrm{e}+04$ & $2.74 \mathrm{e}+04$ & $2.805 e+04$ & $2.805 e+04$ & $2.805 e+04$ \\
\hline
\end{tabular}

TABLE V. RESIDUAL ENERGY VS TIME (SECONDS)

\begin{tabular}{|c|c|c|c|c|c|c|c|c|c|c|c|c|}
\hline \multirow{2}{*}{ Protocol } & \multicolumn{2}{|c|}{ Performance } & \multirow{2}{*}{ 1000s } & \multirow{2}{*}{ 2000s } & \multirow{2}{*}{$3000 \mathrm{~s}$} & \multirow{2}{*}{$4000 \mathrm{~s}$} & \multirow{2}{*}{$5000 \mathrm{~s}$} & \multirow{2}{*}{$6000 \mathrm{~s}$} & \multirow{2}{*}{$7000 \mathrm{~s}$} & \multirow{2}{*}{$8000 s$} & \multirow{2}{*}{$9000 \mathrm{~s}$} & \multirow{2}{*}{$10000 s$} \\
\hline & \%Age & Average & & & & & & & & & & \\
\hline LAEEBA & $100 \%$ & 0.888 & 2.965 & 1.928 & 1.479 & 1.139 & 0.7988 & 0.4586 & 0.1182 & 0 & 0 & 0 \\
\hline DARE & $2.239 \%$ & 1.989 & 4.881 & 4.169 & 3.457 & 2.745 & 2.03 & 1.314 & 0.7188 & 0.3272 & 0.1931 & 0.05909 \\
\hline ACIDS & $1.091 \%$ & 0.969 & 3.281 & 2.569 & 1.857 & 1.147 & 0.5748 & 0.1954 & 0.06131 & 0 & 0 & 0 \\
\hline
\end{tabular}

\section{CONCLUSION \& FUTURE WORK}

Abundant of research papers have been published that focus different parameters like; energy consumption, topology, throughput, QoS, end-to-end delay, stability period. Different researchers proposed protocols to enhance the performance of WBASNS by focusing the above said parameters. In this research thesis we focused to increase the stability period, decrease the end-to-delay, and increase the throughput. In order to achieve our goal we proposed a complete topology with mathematical work. In this research we implement eight sensors on the human with two sinks. The main idea was to implement AnyCasting by using two sink nodes with the concept of AnyCating. In mathematical work, we have also RSSI to select forwarder node in case of tie situation.
We compared our protocol ACIDS with two existing protocol LAEEBA and DARE through Matlab simulator. Before simulation the performance of LAEEBA and DARE have been analyzed. Performance of these three protocols has been analyzed for the individual parameter separately in different time (seconds) started from 1000s to 10000s.

Simulation results shows that the performance of ACIDS is more efficient than LAEEBA and DARE in throughput, stability period and end-to-end delay. The increased efficiency of ACIDS is due to the availability of more than one sink node instead of one. The sensors send their either S1 or S2 in AnyCast manner. The other reason of efficiency in ACIDS is topological factor; in ACIDS majority of the nodes comes in direct range to sink. This leads to send more data in less delay manner. In this research, we also proved that the energy 
consumption of ACIDS comes in tradeoff with above said parameters. We got improvement in above said parameters but not gained better result in energy efficiency. It is proved that ACIDS is better than LAEEBA and not efficient than DARE. The reason is, DARE focused more on energy efficiency and we have focused to improve the said three parameters and got efficient result. The other reason is we introduce RSSI, for which the protocol do more processing and utilize more energy.

In future, our focus will be on energy conservation in this design consideration; and try to implement energy harvesting concept. This concept will improve the performance in great extant. Energy harvesting is a concept where sensors obtain energy from external sources like thermal energy, solar system, wind power etc. Sensors store and use this energy for their processing purpose. As the WBASN have application driven protocols, different applications are introducing in new researches, so we are willing to implement this protocol to new application. Besides the above discussed issues, there are still other challenges existing in WBASNS that are still unresolved. Among them some are; security, data management, scalability and constant monitoring etc.

\section{REFERENCES}

[1] Yousaf, S., Ahmed, S., Akbar, M., Javaid, N., Khan, Z. A., \&Qasim, U. (2014, November). Incremental relay-based co-cestat protocol for wireless body area networks. In Broadband and Wireless Computing, Communication and Applications (BWCCA), 2014 Ninth International Conference on (pp. 113-119). IEEE.

[2] Tauqir, A., Javaid, N., Akram, S., Rao, A., \& Mohammad, S. N. (2013, October). Distance aware relaying energy-efficient: Dare to monitor patients in multi-hop body area sensor networks. In Broadband and Wireless Computing, Communication and Applications (BWCCA), 2013 Eighth International Conference on (pp. 206-213). IEEE.

[3] Nadeem, Q., NadeemJavaid, S. N. Mohammad, M. Y. Khan, SohabSarfraz, and M. Gull. "Simple: Stable increased-throughput multihop protocol for link efficiency in wireless body area networks." In Broadband and Wireless Computing, Communication and Applications (BWCCA), 2013 Eighth International Conference on, pp. 221-226. IEEE, 2013.

[4] Nadeem, A., Hussain, M. A., Owais, O., Salam, A., Iqbal, S., \&Ahsan, K. (2015). Application specific study, analysis and classification of body area wireless sensor network applications. Computer Networks.

[5] Akram, S., Javaid, N., Tauqir, A., Rao, A., \& Mohammad, S. N. (2013, October). THE-FAME: THreshold Based Energy-Efficient FAtigueMEasurement for Wireless Body Area Sensor Networks Using Multiple Sinks. In Broadband and Wireless Computing, Communication and Applications (BWCCA), 2013 Eighth International Conference on (pp. 214-220). IEEE.
[6] Bahanfar, S., Darougaran, L., Kousha, H., \&Babaie, S. (2011). Reliable communication in wireless body area sensor network for health monitoring.arXiv preprint arXiv: 1112.0393.

[7] Khan, Z. A., Rasheed, M. B., Javaid, N., \& Robertson, B. (2014). Effect of Packet Inter-arrival Time on the Energy Consumption of Beacon Enabled MAC Protocol for Body Area Networks. Procedia Computer Science, 32, 579-586.

[8] Ahmed, S., Javaid, N., Akbar, M., Iqbal, A., Khan, Z. A., \&Qasim, U. (2014, May). LAEEBA: Link Aware and Energy Efficient Scheme for Body Area Networks. In Advanced Information Networking and Applications (AINA), 2014 IEEE 28th International Conference on (pp. 435-440). IEEE.

[9] Javaid, N., Abbas, Z., Fareed, M. S., Khan, Z. A., \&Alrajeh, N. (2013). M-ATTEMPT: A new energy-efficient routing protocol for wireless body area sensor networks. Procedia Computer Science, 19, 224-231.

[10] Fazl-e-Hadi, Abid Ali Minhas, " EAA: Energy Aware Anycast Routing in Wireless Sensor Networks", Journal of Engineering and Applied Sciences (JEAS), Vol: 30 No., January-June 2011.

[11] Ahmed, S., Javaid, N., Yousaf, S., Ahmad, A., Sandhu, M. M., Imran, M., ...\&Alrajeh, N. (2015). Co-LAEEBA: Cooperative link aware and energy efficient protocol for wireless body area networks. Computers in Human Behavior.

[12] Dinkar, P., Gulavani, A., Ketkale, S., Kadam, P., \& Dabhade, S. (2013). Remote health monitoring using wireless body area network. International Journal of Engineering and Advanced Technology (IJEAT) ISSN, 2249, 8958.

[13] Kumari, J., \& Prachi. (2015). An Energy Efficient Routing Algorithm for Wireless Body Area Network. IJWMT, 5(5), 56-62. Doi:10.5815/ijwmt.2015.06.

[14] Kaur, H. P., \& Goyal, K. (2015). Cost Based Efficient Routing for Wireless Body Area Networks.

[15] Liu, S., Wei, X., \& Zhao, M. (2016). Routing Design and Simulation of Body Area Network Based on Node Energy Consumption Control Strategy.

[16] Arrobo, G. E., \& Gitlin, R. D. (2011, August). Improving the reliability of wireless body area networks. In Engineering in Medicine and Biology Society, EMBC, 2011 Annual International Conference of the IEEE (pp. 2192-2195). IEEE.

[17] D'Andreagiovanni, F., \& Nardin, A. (2015). Towards the fast and robust optimal design of Wireless Body Area Networks. Applied Soft Computing,37, 971-982.

[18] Xu, J., Liu, W., Lang, F., Zhang, Y., \& Wang, C. (2010). Distance measurement model based on RSSI in WSN. Wireless Sensor Network, 2(08), 606.

[19] Zhou, Y., Sheng, Z., Mahapatra, C., Leung, V. C., \& Servati, P. (2017). Topology design and cross-layer optimization for wireless body sensor networks. Ad Hoc Networks.

[20] Hassan, M. M., Lin, K., Yue, X., \& Wan, J. (2017). A multimedia healthcare data sharing approach through cloud-based body area network. Future Generation Computer Systems, 66, 48-58. 